\title{
Insect herbivory along environmental gradients
}

\author{
Nigel R. Andrew*, Isobel R. Roberts, Sarah J. Hill \\ Centre for Behavioural and Physiological Ecology, Zoology, University of New England, Armidale, Australia; \\ *Corresponding Author: nigel.andrew@une.edu.au
}

Received 15 July 2012; revised 4 September 2012; accepted 15 September 2012

\section{ABSTRACT}

There is a general assumption in the literature that insect herbivory increases towards the tropics, but decreases with increasing altitude. Similar generalities have been identified along other environmental gradients, such as resource, temperature, climatic and biotic gradients. However there is growing evidence in the scientific literature that such generalities are not consistent. This could be due to a number of reasons including the lack of consistency in the way herbivory is assessed such as different methodologies used by researchers, or fundamental differences in leaf damage caused by different types of insect herbivores. Here we assess 61 publications researching insect herbivory along a range of environmental gradients (both biotic and abiotic) and review the methods that researchers have used to collected their data. We found leaf chewing from samples collected in North America dominated the field and most studies assessed herbivory on a single host plant species. Thirty three percent of the studies assessed latitudinal gradients, while $10 \%$ assessed altitudinal gradients. Insect herbivory was most commonly expressed as percentage leaf damage using point herbivory. Fewer studies measured a range of different types of herbivory (such as sap sucking, leaf mining, galling, and root feeding) as leaves aged. From our synthesis, we hope that future research into insect herbivory along environmental gradients will take into account herbivory other than just leaf chewing, such as sap sucking, which may cause more damage to plants. Future research should also assess herbivory as a rate, rather than just a single point in time as damage to a young leaf may be more costly to a plant than damage to a mature or senescing leaf. Measurements of plant traits will also assist in comparing herbivory across habitats, plant species, and within species physiological variation. The true impacts that insects have on plants via herbivory along environmental gradients are still poorly understood.

Keywords: Insect Herbivory; Gradients, Leaf Chewing; Sap Sucking; Galling; Root Feeding

\section{INTRODUCTION}

There is a general assumption in the literature that herbivory increases towards the tropics, but decreases with increasing altitude. However there is growing evidence that such generalities are not consistent across space, time and interacting resource gradients. These inconsistencies may be due to a variety of different methodologies used by researchers, or fundamental differences in leaf damage caused by different types of herbivores. Here we assess the current state of the literature and determine whether the study of insect herbivory along environmental gradients measuring different insect taxa can be compared across space, time and research methodologies. We also examine the biases in the type of herbivory collections that have been undertaken and advocate the use of more consistent methods that can be reproduced in all ecosystems.

Since Coley and Aide published their seminal work finding that herbivory was higher in the tropics compared to the temperate zones [1], there has been a suite of papers citing this research but not actually testing its assumptions. Researchers citing this work have failed to acknowledge the problems of using a small number of sites at each zone or the ability to compare herbivory in one habitat as a surrogate for another at similar latitudes both north and south of the equator. For example, the northern hemisphere is not a mirror image of the southern hemisphere [2] with substantial asymmetry between hemispheres leading to very different plant and animal species and associated interactions.

Another major issue with assessing insect herbivory along environmental gradients is the variation among plant species sampled within the gradient. Different plant species are found across all gradients, especially those covering tropical and temperate zones, lowlands to al- 
pine areas, and coastal to inland desert regions. Along smaller gradients plants have different evolutionary relationships, different interactions with other species, and are under different competitive and symbiotic pressures both within and across species. From a structural point of view, plants have a suite of different aged leaves, with different chemical compositions [3], so measuring leaf herbivory using a “consistent” leaf structure along a gradient can be difficult when morphological and chemical aspects have not been controlled for [4-7].

Here we compare literature that has been published on insect herbivory along a range of gradients (including latitudinal, altitudinal, temperature, successional and architectural among others) and assess the methods that they have used to carry out their herbivory measurements.

\section{METHODS}

In this review we assessed how insect herbivory has been measured across a range of studies. We have collected information on study location, the type of insect herbivory measured, plant species, gradient, and how herbivory was measured, either as a single point in time, or measured as a rate (either over leaf lifespan or between two points in time).

A literature search was conducted in Scopus for literature with the keywords: "herbivory and insect", "herbivory and [each of the insect order names]". Citations and abstracts were downloaded and assessed. Each article was then assessed to determine if it was appropriate for further assessment, and a reprint was downloaded. It is known that Scopus also lists keywords in addition to the author's keywords, with many articles not actually referring to herbivory, in any part of their paper. If herbivory (or equivalent term) was not referred to in the paper (abstract or main body) it was not included in the assessment.

\section{RESULTS AND DISCUSSION}

Of the 61 studies assessed over 30 years between 1982 and 2012 (Table 1), 28 datasets were collected from North America, eight from Europe, six from South America, five from Australia and Central America, three were from multiple regions/worldwide, and one from China. A bias towards North America is common for many research areas, but such a strong dominance was unexpected. This bias in the literature towards northern hemisphere studies is particularly problematic when attempting to make generalisations across space, time and resource gradients. North American temperate climate is very different to that found in the southern hemisphere, so direct comparisons with similar latitudes in the south can be misleading [2]. Northern Hemisphere forests are more deciduous than those in the tropics and the southern hemisphere [8] making direct comparisons of herbivory difficult especially when using different collection and assessment techniques across the plant and insect phylogeny. Plants in deciduous forests will transfer up to $50 \%$ of leaf nitrogen and phosphorous back into the plant [9] seasonally, so insect herbivores such as aphids and sap suckers that can exploit this resource movement will capitalize on this. Chewing herbivory is also reduced by deciduousness with early autumnal coloration onset in the previous season reducing chewing damage in the next growing season [10]. This relatively high publication of studies from North America and Europe is obvious as it is where most researchers who publish in western scientific journals reside and carry out their research. Papers published in languages other than English are biased against in terms of having access to English speaking audiences, and especially Asian research journals were not identified in the literature search. This could be due to a high rejection rate of manuscripts from non native English speaking countries [11], reducing the ability of research from countries such as China and India to be exposed to a wider audience.

In the 61 studies reviewed, the type of herbivory assessed was also biased. Thirty one studies assessed leaf chewing herbivory. Only nine studies assessed sap sucking herbivory, and a further nine studies assessed a range of herbivory types. Six studies did not assess a specific herbivory type and four were reviews. Other studies assessed a range of herbivory types as well as pit, mining and galling herbivory separately. Even though chewing herbivory is the most obvious and easiest type of herbivory to measure, other herbivory types, such as sap-sucking into phloem and xylem can be more damaging to a plant. For example sap-sucking psyllids on Eucalypts in Australia can reduce sap flow by $50 \%$ and reduce leaf mass by $20 \%$ from necrotic tissue loss [12]. In addition, the mirid bug herbivore, Capsodes infuscatus significantly reduced the flower nectar yield of a Mediterranean geophyte, Asphodelus aestivus by $40 \%$ when insect herbivore density treatments were carried out [13]. A metaanalysis based on 52 papers assessing the effects of sap feeding on growth photosynthesis and reproduction of woody plants, compared to folivory (chewing damage) found that sap-feeders impose a more severe overall negative impact on plant performance than do defoliators [14]. By concentrating research efforts on one type of herbivore, particularly chewing herbivores, there may in fact be an under estimation of the true impact of all types of insect herbivory across environmental gradients.

Of the studies assessed, most were carried out on a single tree species $(n=14)$, followed by single native shrub species $(\mathrm{n}=11)$, multiple native tree species $(\mathrm{n}=$ 9 ), single herb/grass species $(n=8)$, multiple native 
Table 1. Review of literature assessing herbivory across a range of environmental gradients. Table includes region were data was collected, herbivory type assessed, number of plant species that data was collected from, gradient assessed, how herbivory was measured, and if it was a rate or point measurement, with major findings of the paper that were directly related to herbivory.

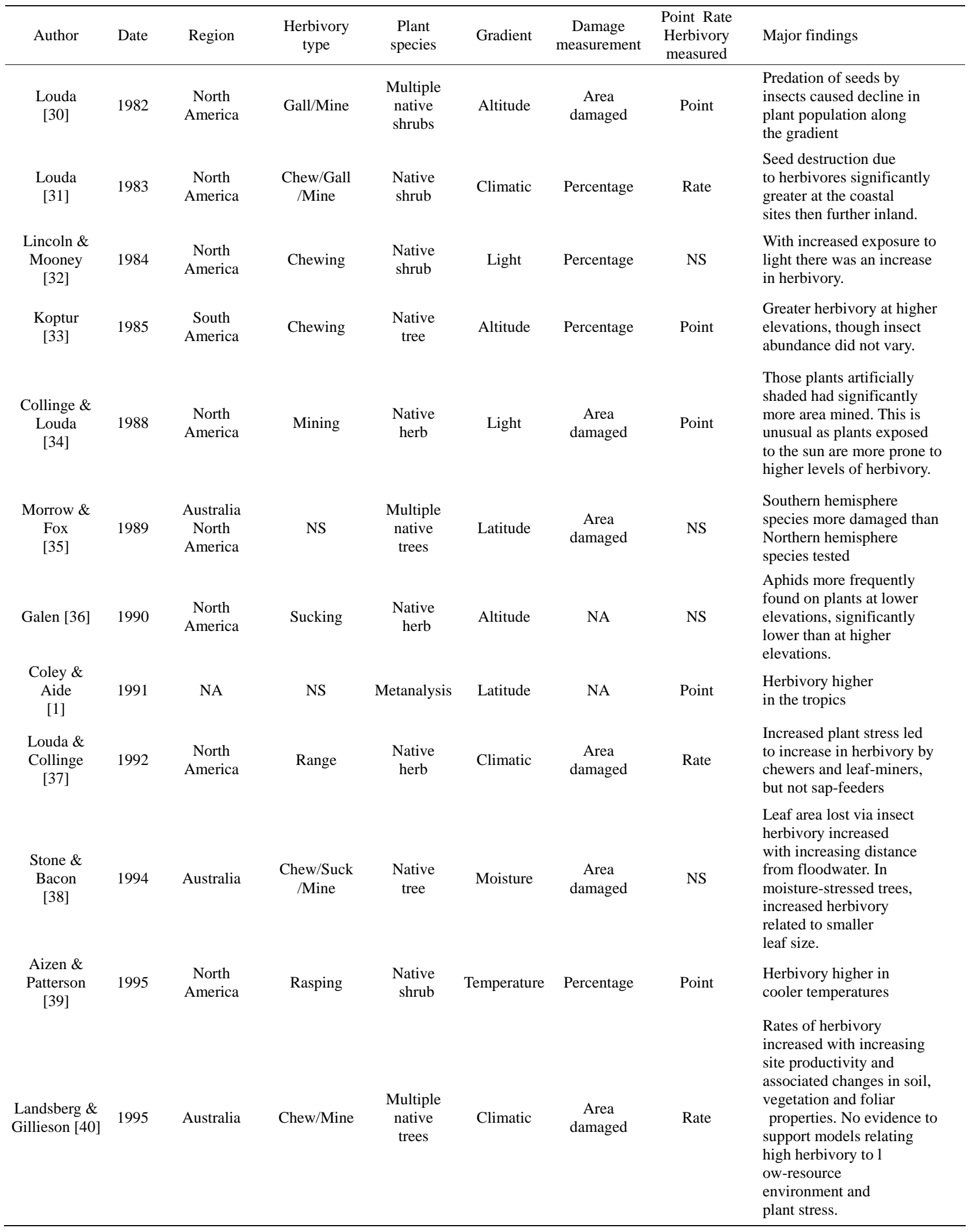


Continued

\begin{tabular}{|c|c|c|c|c|c|c|c|c|}
\hline $\begin{array}{l}\text { Louda \& } \\
\text { Rodman } \\
\quad[41]\end{array}$ & 1996 & $\begin{array}{c}\text { North } \\
\text { America }\end{array}$ & $\begin{array}{l}\text { Chew/Suck } \\
\text { /Mine }\end{array}$ & $\begin{array}{l}\text { Native } \\
\text { herb }\end{array}$ & Temperature & $\begin{array}{c}\text { Area } \\
\text { damaged }\end{array}$ & Rate & $\begin{array}{l}\text { Shade removal increased } \\
\text { herbivory. Insect abundances } \\
\text { higher in the sun. }\end{array}$ \\
\hline $\begin{array}{l}\text { Kelly } \\
{[43]}\end{array}$ & 1998 & $\begin{array}{c}\text { North } \\
\text { America }\end{array}$ & Sucking & $\begin{array}{l}\text { Native } \\
\text { shrub }\end{array}$ & Altitude & NA & NS & $\begin{array}{l}\text { At higher elevations there } \\
\text { were fewer flowering } \\
\text { shoots infested with aphids. }\end{array}$ \\
\hline $\begin{array}{c}\text { Uriarte \& } \\
\text { Schmitz } \\
\text { [44] }\end{array}$ & 1998 & $\begin{array}{c}\text { North } \\
\text { America }\end{array}$ & Chew/Suck & $\begin{array}{l}\text { Native } \\
\text { shrub }\end{array}$ & Succession & $\begin{array}{c}\text { Area } \\
\text { damaged }\end{array}$ & Rate & $\begin{array}{l}\text { Herbivores only decreased } \\
\text { goldenrod shoot biomass in } \\
\text { the late successional stage. }\end{array}$ \\
\hline $\begin{array}{c}\text { Maron } \\
\text { [45] }\end{array}$ & 1998 & $\begin{array}{c}\text { North } \\
\text { America }\end{array}$ & Chew/Roots & $\begin{array}{l}\text { Native } \\
\text { shrub }\end{array}$ & $\begin{array}{c}\text { Tree } \\
\text { architecture }\end{array}$ & $\begin{array}{c}\text { Area } \\
\text { damaged }\end{array}$ & Point & $\begin{array}{l}\text { No significant interaction } \\
\text { between herbivory above } \\
\text { or below ground. }\end{array}$ \\
\hline $\begin{array}{c}\text { McEvoy \& } \\
\text { Coombs [46] }\end{array}$ & 1999 & Worldwide & Range & $\begin{array}{l}\text { Native } \\
\text { herb }\end{array}$ & Disturbance & Modelling & NS & $\begin{array}{l}\text { Herbivory by insects } \\
\text { controlled ragwort when } \\
\text { habitat was disturbed. }\end{array}$ \\
\hline $\begin{array}{c}\text { Alonso } \\
\text { [47] }\end{array}$ & 1999 & Europe & Chewing & $\begin{array}{l}\text { Native } \\
\text { tree }\end{array}$ & Altitude & $\begin{array}{c}\text { Area } \\
\text { damaged }\end{array}$ & Rate & $\begin{array}{l}\text { Increased in herbivory } \\
\text { in warmer areas closes } \\
\text { to sea level. }\end{array}$ \\
\hline $\begin{array}{l}\text { Fagan \& } \\
\text { Bishop } \\
\text { [48] }\end{array}$ & 2000 & $\begin{array}{c}\text { North } \\
\text { America }\end{array}$ & NS & $\begin{array}{l}\text { Native } \\
\text { herb }\end{array}$ & Succession & Percentage & Rate & $\begin{array}{l}\text { Removing insect herbivores } \\
\text { increasedgrowth of lupine } \\
\text { at the edge region, but } \\
\text { not in the core region. }\end{array}$ \\
\hline $\begin{array}{l}\text { Pennings } \\
\text { et al. } \\
\text { [49] }\end{array}$ & 2001 & $\begin{array}{l}\text { North } \\
\text { America }\end{array}$ & Chewing & $\begin{array}{l}\text { Multiple } \\
\text { native } \\
\text { shrubs }\end{array}$ & Latitude & $\begin{array}{c}\text { Area } \\
\text { damaged }\end{array}$ & Rate & $\begin{array}{l}\text { Evidence of latitudinal } \\
\text { gradient in plant palatability, } \\
\text { with herbivores showing a } \\
\text { significant or marginally } \\
\text { significant preference } \\
\text { for northern plants. }\end{array}$ \\
\hline Rand [50] & 2002 & $\begin{array}{l}\text { North } \\
\text { America }\end{array}$ & Chewing & $\begin{array}{l}\text { Native } \\
\text { shrub }\end{array}$ & Salinity & Percentage & Rate & $\begin{array}{l}\text { Herbivory highest } \\
\text { at mid-levels. }\end{array}$ \\
\hline $\begin{array}{l}\text { Bale } \\
\text { et al. [51] }\end{array}$ & 2002 & N/A & Review & Review & Temperature & NA & NS & $\begin{array}{l}\text { Intensity of herbivory } \\
\text { increases with rising } \\
\text { temperatures at constant } \\
\text { latitude. }\end{array}$ \\
\hline $\begin{array}{l}\text { Freeman } \\
\text { et al. [52] }\end{array}$ & 2003 & $\begin{array}{c}\text { North } \\
\text { America }\end{array}$ & Chewing & $\begin{array}{r}\text { Native } \\
\text { shrub }\end{array}$ & Altitude & $\begin{array}{c}\text { Area } \\
\text { damaged }\end{array}$ & Point & $\begin{array}{l}\text { Seed predation was lower } \\
\text { on clipped (artificial browsing) } \\
\text { plants at higher } \\
\text { elevations as clipping } \\
\text { delayed blooming and } \\
\text { reduced the length of } \\
\text { the growing season. }\end{array}$ \\
\hline $\begin{array}{c}\text { Hodar \& } \\
\text { Zamora } \\
\text { [53] }\end{array}$ & 2004 & Europe & Chewing & $\begin{array}{l}\text { Native } \\
\text { tree }\end{array}$ & Altitude & Percentage & Rate & $\begin{array}{l}\text { Defoliation intensity } \\
\text { shows a significant } \\
\text { association with previous } \\
\text { warm winters. }\end{array}$ \\
\hline $\begin{array}{c}\text { Cuevas-Reyes } \\
{[54]}\end{array}$ & 2004 & $\begin{array}{l}\text { Central } \\
\text { America }\end{array}$ & Galling & $\begin{array}{l}\text { Multiple } \\
\text { trees } \\
\text { and } \\
\text { shrubs }\end{array}$ & Soil fertility & Galling & NS & $\begin{array}{l}\text { Low frequency and density } \\
\text { of galling insects on } \\
\text { fertile soils }\end{array}$ \\
\hline $\begin{array}{l}\text { Gaston } \\
\text { et al. } \\
\text { [55] }\end{array}$ & 2004 & Europe & Mining & $\begin{array}{l}\text { Native } \\
\text { shrub }\end{array}$ & Latitude & NA & NS & $\begin{array}{l}\text { Population dynamics } \\
\text { of holly leaf-miner } \\
\text { across its geographical } \\
\text { range is complex. } \\
\text { Mortality relates to varying } \\
\text { host plant quality. }\end{array}$ \\
\hline $\begin{array}{l}\text { Andrew \& } \\
\text { Hughes } \\
\text { [22] }\end{array}$ & 2005 & Australia & $\begin{array}{l}\text { Chew/Suck } \\
\text { /Mine }\end{array}$ & $\begin{array}{l}\text { Native } \\
\text { shrub }\end{array}$ & Latitude & Percentage & Rate & $\begin{array}{l}\text { No significant difference } \\
\text { across gradient. }\end{array}$ \\
\hline
\end{tabular}




\section{Continued}

\begin{tabular}{|c|c|c|c|c|c|c|c|c|}
\hline $\begin{array}{l}\text { Novotny \& } \\
\text { Basset } \\
\text { [56] }\end{array}$ & 2005 & NA & Review & Review & Latitude & NA & NS & $\begin{array}{l}\text { Host specificity between } \\
\text { temperate and tropical } \\
\text { forests are difficult to } \\
\text { assess due to differences in } \\
\text { collection methods. }\end{array}$ \\
\hline $\begin{array}{l}\text { Knight \& Holt } \\
\text { [57] }\end{array}$ & 2005 & $\begin{array}{l}\text { North } \\
\text { America }\end{array}$ & Pit feed & $\begin{array}{l}\text { Multiple } \\
\text { trees } \\
\text { and } \\
\text { shrubs }\end{array}$ & Disturbance & Percentage & Rate & $\begin{array}{l}\text { Insect abundance at the } \\
\text { edge of burned areas, } \\
\text { greater than in the interior. } \\
\text { Herbivory greater at } \\
\text { edge than in the interior of } \\
\text { burned sites. }\end{array}$ \\
\hline $\begin{array}{l}\text { Pennings \& } \\
\text { Silliman } \\
\text { [58] }\end{array}$ & 2005 & $\begin{array}{l}\text { North } \\
\text { America }\end{array}$ & Chewing & $\begin{array}{l}\text { Multiple } \\
\text { native } \\
\text { shrubs }\end{array}$ & Succession & Modelling & Point & $\begin{array}{l}\text { High latitude plants more } \\
\text { palatable to herbivores } \\
\text { than low latitude plants. } \\
\text { Low latitude plants } \\
\text { experienced greater levels } \\
\text { of leaf damage from } \\
\text { consumers } \\
\text { than did high latitude plants. } \\
\text { If herbivores introduced } \\
\text { early on during the invasion } \\
\text { of a pest species, they } \\
\text { can act as an effective } \\
\text { biocontrol agent, with } \\
\text { the model suggesting that } \\
\text { the more established plants } \\
\text { are fed on less extremely. }\end{array}$ \\
\hline $\begin{array}{l}\text { Unsicker } \\
\text { et al. [60] }\end{array}$ & 2006 & Europe & Chewing & $\begin{array}{l}\text { Multiple } \\
\text { native } \\
\text { shrubs }\end{array}$ & $\begin{array}{l}\text { Plant } \\
\text { species } \\
\text { richness }\end{array}$ & $\begin{array}{c}\text { Area } \\
\text { damaged }\end{array}$ & Point & $\begin{array}{l}\text { Mean herbivory levels } \\
\text { correlated negatively } \\
\text { with plant species richness }\end{array}$ \\
\hline $\begin{array}{l}\text { Maron \& } \\
\text { Crone [62] }\end{array}$ & 2006 & $\begin{array}{l}\text { North } \\
\text { America }\end{array}$ & Review & $\begin{array}{l}\text { Native } \\
\text { tree }\end{array}$ & Succession & $\begin{array}{c}\text { Area } \\
\text { damaged }\end{array}$ & Point & $\begin{array}{l}\text { Tallow trees that had } \\
\text { been growing for the } \\
\text { longest had lower levels } \\
\text { of herbivory. } \\
\text { Noted that there is variation } \\
\text { in the "magnitude of herbivory" } \\
\text { across a variety of } \\
\text { environmental gradients, } \\
\text { though claim most } \\
\text { studies needed to be more ro- } \\
\text { bust. }\end{array}$ \\
\hline $\begin{array}{l}\text { Pennings } \\
\text { et al. [63] }\end{array}$ & 2007 & Europe & Chewing & $\begin{array}{l}\text { Multiple } \\
\text { native } \\
\text { shrubs }\end{array}$ & Latitude & Percentage & Point & $\begin{array}{l}\text { High latitude plants more } \\
\text { palatable to herbivores than } \\
\text { low latitude plants. Plants } \\
\text { at low latitude field sites } \\
\text { experience greater levels } \\
\text { of herbivore pressure than } \\
\text { plants at high latitude sites. }\end{array}$ \\
\hline $\begin{array}{c}\text { Andrew \& } \\
\text { Hughes } \\
\text { [24] }\end{array}$ & 2007 & Australia & $\begin{array}{l}\text { Chew/Suck } \\
\text { /Mine }\end{array}$ & $\begin{array}{l}\text { Multiple } \\
\text { trees } \\
\text { and } \\
\text { shrubs }\end{array}$ & Latitude & Percentage & Rate & $\begin{array}{l}\text { No significant difference } \\
\text { across gradient. }\end{array}$ \\
\hline $\begin{array}{l}\text { Pontes } \\
\text { Ribiero \& } \\
\text { Basset } \\
\text { [64] }\end{array}$ & 2007 & $\begin{array}{l}\text { Central } \\
\text { America }\end{array}$ & Chew/Gall & $\begin{array}{l}\text { Native } \\
\text { tree }\end{array}$ & $\begin{array}{c}\text { Tree } \\
\text { architecture }\end{array}$ & Percentage & Point & $\begin{array}{l}\text { Free living herbivory } \\
\text { decreases with height, } \\
\text { gall diversity increases } \\
\text { with height. }\end{array}$ \\
\hline $\begin{array}{c}\text { Ribeiro \& } \\
\text { Basset [64] }\end{array}$ & 2007 & $\begin{array}{l}\text { Central } \\
\text { America }\end{array}$ & Chew/Gall & $\begin{array}{l}\text { Multiple } \\
\text { trees } \\
\text { and } \\
\text { shrubs }\end{array}$ & $\begin{array}{c}\text { Tree } \\
\text { architecture }\end{array}$ & Percentage & Point & $\begin{array}{l}\text { Leaf herbivory decreased } \\
\text { with height, galling } \\
\text { increased with height. }\end{array}$ \\
\hline $\begin{array}{c}\text { Kozlov } \\
\text { [65] }\end{array}$ & 2008 & Europe & Chew/Mine & $\begin{array}{l}\text { Native } \\
\text { tree }\end{array}$ & Latitude & Percentage & Point & $\begin{array}{l}\text { Leaf damage decreased } \\
\text { with latitude. }\end{array}$ \\
\hline $\begin{array}{l}\text { Sinclair \& } \\
\text { Hughes } \\
\text { [66] }\end{array}$ & 2008 & Australia & Mining & $\begin{array}{l}\text { Multiple } \\
\text { native } \\
\text { trees }\end{array}$ & Rainfall & NA & Point & $\begin{array}{l}\text { No significant difference } \\
\text { across gradient. }\end{array}$ \\
\hline
\end{tabular}


Continued

\begin{tabular}{|c|c|c|c|c|c|c|c|c|}
\hline $\begin{array}{l}\text { Wolf } \\
\text { et al. } \\
{[67]}\end{array}$ & 2008 & Europe & Chewing & $\begin{array}{l}\text { Native } \\
\text { tree }\end{array}$ & $\begin{array}{l}\text { Latitude/ } \\
\text { Altitude }\end{array}$ & Modelling & Point & $\begin{array}{l}\text { A decrease of insect impact } \\
\text { towards the north and higher } \\
\text { up in the mountains. }\end{array}$ \\
\hline $\begin{array}{l}\text { Adams \& } \\
\text { Zhang } \\
\text { [68] }\end{array}$ & 2009 & $\begin{array}{l}\text { North } \\
\text { America }\end{array}$ & Range & $\begin{array}{l}\text { Multiple } \\
\text { native } \\
\text { trees }\end{array}$ & Latitude & Percentage & Point & $\begin{array}{l}\text { Less percentage area damage } \\
\text { per leaf in lower latitude } \\
\text { areas of eastern North Amer- } \\
\text { ica. }\end{array}$ \\
\hline $\begin{array}{l}\text { Adams et al. } \\
\text { [69] }\end{array}$ & 2009 & $\begin{array}{l}\text { North } \\
\text { America }\end{array}$ & Range & $\begin{array}{l}\text { Multiple } \\
\text { native } \\
\text { trees }\end{array}$ & Latitude & Percentage & Point & $\begin{array}{l}\text { No significant latitudinal } \\
\text { trend in herbivory, condensed } \\
\text { and } \\
\text { hydrolyzable tannins, } \\
\text { or total phenolics. Findings } \\
\text { contradict the theory } \\
\text { that low latitude plants are } \\
\text { better defended. }\end{array}$ \\
\hline $\begin{array}{l}\text { Adams } \\
\text { et al. [69] }\end{array}$ & 2009 & $\begin{array}{c}\text { North } \\
\text { America/Asia }\end{array}$ & Range & $\begin{array}{l}\text { Multiple } \\
\text { native } \\
\text { trees }\end{array}$ & Latitude & $\begin{array}{c}\text { Area } \\
\text { damaged }\end{array}$ & NS & $\begin{array}{l}\text { Lowland tropical forest } \\
\text { has significantly } \\
\text { higher leaf herbivory } \\
\text { than temperate forest. } \\
\text { Used different index, } \\
\text { which suggested different } \\
\text { results-Percent damage "per } \\
\text { unit time of growing } \\
\text { season" is calculated } \\
\text { based on an estimate of leaf } \\
\text { lifetime, tropical lowland } \\
\text { herbivory damage is less } \\
\text { than temperate zone. } \\
\text { Intensity of herbivory on } \\
\text { leaves per unit of time, } \\
\text { the opposite trend occurs. }\end{array}$ \\
\hline $\begin{array}{l}\text { Sobek } \\
\text { et al. [71] }\end{array}$ & 2009 & Europe & $\begin{array}{l}\text { Chew/Mine } \\
\text { /Gall }\end{array}$ & $\begin{array}{l}\text { Multiple } \\
\text { native } \\
\text { trees }\end{array}$ & $\begin{array}{l}\text { Plant } \\
\text { species } \\
\text { richness }\end{array}$ & Percentage & Point & $\begin{array}{l}\text { Only one species (Beech) } \\
\text { showed a decline in damage } \\
\text { due to leaf-chewing } \\
\text { herbivores across the tree } \\
\text { diversity gradient. No } \\
\text { significant patterns for } \\
\text { galls and mines. }\end{array}$ \\
\hline $\begin{array}{l}\text { Miller et al. } \\
\quad[72]\end{array}$ & 2009 & $\begin{array}{l}\text { North } \\
\text { America }\end{array}$ & $\begin{array}{l}\text { Chew/Suck } \\
\text { /Mine }\end{array}$ & $\begin{array}{l}\text { Native } \\
\text { shrub }\end{array}$ & Altitude & Percentage & Rate & $\begin{array}{l}\text { Insect abundance and } \\
\text { damage by insects } \\
\text { decreased with increasing } \\
\text { elevation. }\end{array}$ \\
\hline $\begin{array}{l}\text { del-Val \& } \\
\text { Armesto } \\
\text { [73] }\end{array}$ & 2010 & $\begin{array}{l}\text { South } \\
\text { America }\end{array}$ & NS & $\begin{array}{l}\text { Native } \\
\text { tree }\end{array}$ & Latitude & Percentage & Rate & $\begin{array}{l}\text { Invertebrate herbivory } \\
\text { greaterin temperate forests. }\end{array}$ \\
\hline $\begin{array}{l}\text { O’Neill } \\
\text { et al. [74] }\end{array}$ & 2010 & $\begin{array}{l}\text { North } \\
\text { America }\end{array}$ & $\begin{array}{l}\text { Chew/Suck } \\
\text { /Skel }\end{array}$ & $\begin{array}{l}\text { Agricultural } \\
\text { species }\end{array}$ & $\mathrm{CO}_{2}$ & Variable & NS & $\begin{array}{l}\text { Concentration of plant } \\
\text { compoundshigher in } \\
\text { plants grown under } \\
\text { elevated levels of } \mathrm{CO}_{2} \text {. } \\
\text { Leaf skeletonisors the main } \\
\text { cause of leaf damage. }\end{array}$ \\
\hline $\begin{array}{l}\text { Schuldt } \\
\text { et al. [75] }\end{array}$ & 2010 & China & $\begin{array}{l}\text { Chew/Mine } \\
\text { /Gall }\end{array}$ & $\begin{array}{l}\text { Multiple } \\
\text { trees and } \\
\text { shrubs }\end{array}$ & $\begin{array}{l}\text { Plant Sp } \\
\text { Rich }\end{array}$ & $\begin{array}{c}\text { Area } \\
\text { damaged }\end{array}$ & Point & $\begin{array}{l}\text { Herbivory was greater } \\
\text { with greater plant diversity. }\end{array}$ \\
\hline $\begin{array}{l}\text { Ballhorn } \\
\text { et al. } \\
\text { [76] }\end{array}$ & 2011 & $\begin{array}{l}\text { Central } \\
\text { America }\end{array}$ & Chewing & $\begin{array}{l}\text { Multiple } \\
\text { native } \\
\text { shrubs }\end{array}$ & $\begin{array}{l}\mathrm{CO}_{2} / \text { Leaf } \\
\text { age }\end{array}$ & $\begin{array}{c}\text { Area } \\
\text { damaged }\end{array}$ & Point & $\begin{array}{l}\text { Highest herbivore damage } \\
\text { in mature leaves under } \\
\text { natural } \mathrm{CO}_{2} \text { to highest damage } \\
\text { of young leaves } \\
\text { under elevated } \mathrm{CO}_{2}\end{array}$ \\
\hline
\end{tabular}




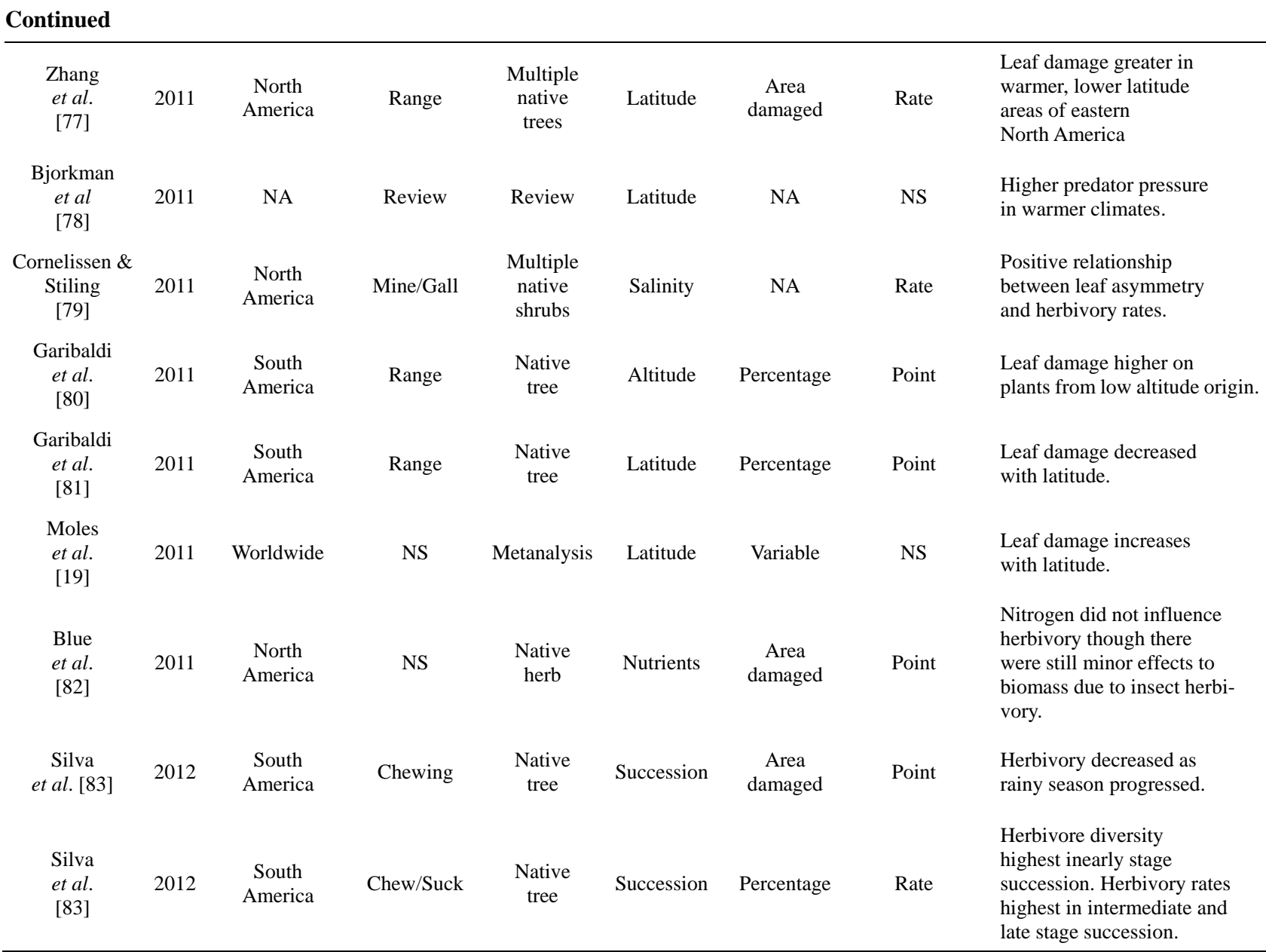

shrub species $(\mathrm{n}=7)$ or multiple native tree/shrub species $(n=5)$. For studies along gradients, restricting the analyses to a single host plant species can be problematic as the gradient length is also constrained by the host plant distribution, but it is the simplest way to constrain host plant variables between species. However, even within host plant species, leaf chemistry and physiological traits can be substantially different. These differences may include variation across plant genotypes and geographical ranges, leaf chemical and physiological variation across different aged leaves, localized soil and microclimatic conditions, and previous herbivore feeding on plant tissue and plant viruses [15-18]. Assessing traits enables direct analysis of herbivory in relation to not only a range of plants, but to leaves at different ages and plants at different ages. For example Moles et al. [19] assessed a range of plant traits and herbivory across 301 plant species from 75 sites world-wide. These traits included leaf toughness and size, polyethylene glycolbinding capacity, nitrogen digestibility, lipid concentration, ash content, force of fracture, specific leaf area, C:N ratio, delayed greening, spines, hair on mature leaves, and cyanogenesis. Therefore assessing plant trait differ- ences along gradients would be much more informative [20], and measurements across large scale gradients at multiple sites could be more comparable.

Insect herbivory along latitudinal gradients were the most studied environmental gradient $(n=20)$ followed by altitudinal gradients $(\mathrm{n}=10)$, with 6 studies along successional gradients, and three along climatic, and host plant architectural gradients. Coley and Aide's seminal 1991 paper [1] was one of the crucial synthesis of information to give future researchers the testable hypotheses that there is more herbivory in the tropics versus the temperate zones. It is now also becoming recognized from trends in the literature that altitudinal gradients are not just smaller scale latitudinal gradients-both have their own unique differences, and thus are both equally important to assess. Climatic gradients are also becoming a more useful way to assess potential impacts of climate change [21]. They incorporate both variation in temperature and rainfall, rather than just assessing changes in herbivory along latitudinal gradients [22] which is usually a surrogate for changes in temperature and is particularly important for understanding the impacts of climate change on insect/plant interactions. 
Percentage damage of leaf tissue was more widely measured than amount of leaf tissue damage per size of leaf (24 studies versus 19 studies). By using percentage herbivory data, researchers commonly arcsin transform their data to "normalize" it for standard univariate statistical analysis. This analysis of proportional data after arcsine square root transformation should not be used [23]. Instead, logistic regression and logit transformation of actual tissue area damaged (normalized by total leaf area) has greater interpretability and higher power than proportional data that has been arcsin transformed [23] and enables data to be analysed and interpreted correctly.

Assessing herbivory from leaf emergence to leaf senescence can be difficult to compare changes along gradients. Here we found 27 studies used point herbivory, while 18 used rate of herbivory, and 16 studies were nonspecific in how herbivory was assessed. The importance of using consistent methods can be illustrated by Andrew and Hughes [22]. In 2005, they did the first broad-scale test for a latitudinal gradient in herbivory made with consistent methods, in similar habitat type, over the entire lifespan of leaves (phyllodes). They assessed different types of leaf damage (chewing, sap-sucking and mining herbivory) along the entire coastal latitudinal range of a single host plant, Acacia falcata. Even though the range was relatively short $(1150 \mathrm{~km})$ it did go from a temperate zone into a sub-tropical zone of Australia. With these consistent methods they found no significant differences in the rate of herbivory among latitudes sampled. Andrew and Hughes also assessed herbivory along this gradient and compared it to herbivory on the same host plant transplanted to a warmer climate [24] and found that rates of herbivory did not significantly differ between the transplanted individuals and plants endemic to the sites within the natural range. This suggests that the methods used to compare herbivory along gradients, whether they be latitudinal, altitudinal, climatic, or environmental, need to be consistent, and assessing the rate of herbivory is crucial to such comparisons [25]. Low-man [25] found that rates of herbivory were fourfold higher when using herbivory assessed over time compared to point sampled herbivory demonstrating that rates of herbivory were far more informative than simply measuring herbivory as a snap-shot in time.

Insect herbivory was most commonly assessed as leaf chewing herbivory at a single point in time (Table 1). The amount of leaf tissue missing was measured as obvious leaf feeding damage with most studies not recording a range of important observations relating to true damage to the plant. Leaf longevity of evergreen tree species ranges from under 100 days to over 12 years [4-6] and this has huge consequences for plant investment into leaf tissue. There is a trade-off between leaf longevity, construction costs for the plant producing the leaf, and photosynthetic rates [7]. Measurement of leaf damage by insect herbivores should consider leaf age and lifespan. This can only be done via measuring rates of herbivory across the leaf lifespan, and not done via point assessments, unless the leaves are deciduous and the leaf lifespan is easily calculated.

General trends in how insect herbivory may change along biotic or abiotic gradients for different trophic groups is still unclear. For classic gradients, such as those done at different latitudes, the method used to assess herbivory plays a crucial role in the results found. The amount of herbivory has generally been reported as being higher in the tropics than at more temperate latitudes $[15,26]$. Coley and Aide's [1] and Coley and Barone's [26] generalizations have been widely accepted (Coley and Barone paper cited 525 times as of April 2012: Scopus database). However, these generalizations are based on literature compilations comparing studies that used a variety of methodologies, performed under a range of environmental conditions, and using plant species from a variety of phylogenies with different chemical and mechanical properties $[1,27,28]$. The type of herbivory measured also plays a particularly important role, for example leaf chewing herbivores will have very different impacts to sap suckers, gallers and leaf miners. Leaf age will also play an important role in determining the herbivore damage seen on plants.

\section{CONCLUSION}

Most studies assessing insect herbivory along environmental gradients have concentrated on latitudinal gradients with a bias toward chewing herbivores in the Northern Hemisphere. Future research into insect herbivory along gradients should assess herbivory caused by different types of herbivores rather than concentrate on chewers which are much easier to assess in the field but do not give a complete picture with regard to the impact of total insect herbivory on plant species. Future studies should also assess herbivory as a rate, rather than just a single point in time. This could be done by assessing herbivory across the leaf lifespan, or as a rate of herbivory that can be directly compared between host plant species and sample sites. Ideally knowing leaf life span and measuring herbivory at known leaf ages is also important, particularly for leaves that live for more than a single growing season. Assessing herbivory in relation to plant and leaf trait changes is also crucial as this will allow within and between species comparisons across a variety of environmental gradients. Plant traits (morphological and chemical) can act as defences against herbivores both by reducing herbivore performance directly and attracting natural enemies [29]. Such incorporation of more specific and comparable functional plants traits 
and how they change with different types of herbivory will become invaluable in assessing if generalizations can be made to assess insect herbivory across environmental gradients.

\section{REFERENCES}

[1] Coley, P.D. and Aide, T.M. (1991) Comparison of herbivory and plant defences in temperate and tropical broadleaved forests, in plant-animal interactions: Evolutionary ecology in the tropical and temperate regions. John Wiley \& Sons Ltd., Brisbane.

[2] Chown, S.L., Sinclair, B.J., Leinaas, H.P. and Gaston, K.J. (2004) Hemispheric asymmetries in biodiversity-A serious matter for ecology. PLoS Biology, 2, 1701-1707. doi:10.1371/journal.pbio.0020406

[3] Reich, P.B., Walters, M.B. and Ellsworth, D.S. (1992) Leaf life-span in relation to leaf, plant, and stand characteristics among diverse ecosystems. Ecological Monographs, 62, 365-392. doi: $10.2307 / 2937116$

[4] Kikuzawa, K. and Ackerly, D. (1999) Significance of leaf longevity in plants. Plant Species Biology, 14, 39-45. doi:10.1046/j.1442-1984.1999.00005.x

[5] Coley, P.D. (1983) Herbivory and defensive characteristics of tree species in a lowland tropical forest. Ecological Monographs, 53, 209-233. doi:10.2307/1942495

[6] Lowman, M.D. (1992) Leaf growth dynamics and herbivory in five species of Australian rain-forest canopy trees. Journal of Ecology, 80, 433-447. doi:10.2307/2260689

[7] Shipley, B., Lechowicz, M.J., Wright, I. and Reich, P.B. (2006) Fundamental trade-offs generating the worldwide leaf economics spectrum. Ecology, 87, 535-541. doi:10.1890/05-1051

[8] Williams, C.E. (2007) Deciduous forest. In: Robbins, P., Ed., The Encyclopedia of Environment and Society, SAGE Publications: Thousand Oaks, California, 416-417. doi: 10.4135/9781412953924.n273

[9] Marchin, R., Zeng, H. and Hoffmann, W. (2010) Droughtdeciduous behavior reduces nutrient losses from temperate deciduous trees under severe drought. Oecologia, 163, 845-854. doi:10.1007/s00442-010-1614-4

[10] Hagen, S.B., Folstad, I. and Jakobsen, S.W. (2003) Autumn colouration and herbivore resistance in mountain birch (Betula pubescens). Ecology Letters, 6, 807-811. doi:10.1046/j.1461-0248.2003.00496.x

[11] Primack, R.B. and Marrs, R. (2008) Bias in the review process. Biological Conservation, 141, 2919-2920. doi:10.1016/j.biocon.2008.09.016

[12] Cunningham, S., Pullen, K. and Colloff, M. (2009) Wholetree sap flow is substantially diminished by leaf herb ivory. Oecologia, 158, 633-640. doi:10.1007/s00442-008-1170-3

[13] Samocha, Y. and Sternberg, M. (2010) Herbivory by sucking mirid bugs can reduce nectar production in Aphodelus aestivus Brot. Arthropod-Plant Interactions, 4, 153-158. doi:10.1007/s11829-010-9091-6

[14] Zvereva, E.L., Lanta, V. and Kozlov, M.V. (2010) Effects of sap-feeding insect herbivores on growth and reproduction of woody plants: A meta-analysis of experimental studies. Oecologia, 163, 949-960. doi:10.1007/s00442-010-1633-1

[15] Close, D. and Beadle, C. (2003) The ecophysiology of foliar anthocyanin. The Botanical Review, 69, 149-161. doi:10.1663/0006-8101(2003)069[0149:TEOFA]2.0.CO; $\underline{2}$

[16] Külheim, C., Yeoh, S.H., Wallis, I.R., Laffan, S., Moran, G.F. and Foley, W.J. (2011) The molecular basis of quantitative variation in foliar secondary metabolites in Eucalyptus globulus. New Phytologist, 191, 1041-1053. doi:10.1111/j.1469-8137.2011.03769.x

[17] Maschinski, J. and Whitham, T.G. (1989) The continuum of plant responses to herbivory: The influence of plant association, nutrient availability, and timing. The American Naturalist, 134, 1-19. doi:10.1086/284962

[18] Renwick, J.A.A., Zhang, W., Haribal, M., Attygalle, A.B. and Lopez K.D. (2001) Dual chemical barriers protect a plant against different larval stages of an insect. Journal of Chemical Ecology, 27, 1575-1583. doi:10.1023/A:1010402107427

[19] Moles, A.T., Wallis, I.R., Foley, W.J., Warton, D.I., Stegen, J.C., Bisigato, A.J., Cella-Pizarro, L., Clark, C.J., Cohen, P.S., Cornwell, W.K., Edwards, W., Ejrnæs, R., Gonzales-Ojeda, T., Graae, B.J., Hay, G., Lumbwe, F.C., Magaña-Rodríguez, B., Moore, B.D., Peri, P.L., Poulsen, J.R., Veldtman, R., Zeipel, H.Von., Andrew, N.R., Boulter, S.L., Borer, E.T., Campón, F.F., Coll, M., Farji-Brener, A.G., De Gabriel, J., Jurado, E., Kyhn, L.A., Low, B., Mulder, C.P.H., Reardon-Smith, K., Rodríguez-Velázquez, J., Seabloom, E.W., Vesk, P.A., Van Cauter, A., Waldram, M.S., Zheng, Z., Blendinger, P.G., Enquist, B.J., Facelli, J.M., Knight, T., Majer, J.D., Martínez-Ramos, M., McQuillan, P. and Prior, L.D. (2011) Putting plant resistance traits on the map: A test of the idea that plants are better defended at lower latitudes. New Phytologist, 191, 777-788. doi:10.1111/j.1469-8137.2011.03732.x

[20] Moles, A.T., Bonser, S.P., Poore, A.G.B., Wallis, I.R. and Foley, W.J. (2011) Assessing the evidence for latitudinal gradients in plant defence and herbivory. Functional Ecology, 25, 380-388. doi:10.1111/j.1365-2435.2010.01814.X

[21] Bairstow, K.A., Clarke, K.L., McGeoch, M.A. and Andrew, N.R. (2010) Leaf miner and plant galler species richness on Acacia: Relative importance of plant traits and climate. Oecologia, 163, 437-448. doi:10.1007/s00442-010-1606-4

[22] Andrew, N.R. and Hughes, L. (2005) Herbivore damage along a latitudinal gradient: Relative impacts of different feeding guilds. Oikos, 108, 176-182. doi:10.1111/j.0030-1299.2005.13457.x

[23] Warton, D.I. and Hui, F.K.C. (2011) The arcsine is asinine: The analysis of proportions in ecology. Ecology, 92, 3-10. doi:10.1890/10-0340.1

[24] Andrew, N.R. and Hughes, L. (2007) Potential host colonization by insect herbivores in a warmer climate: A transplant experiment. Global Change Biology, 13, 1539-1549. doi:10.1111/j.1365-2486.2007.01393.x

[25] Lowman, M.D. (1992) Herbivory in Australian rainfor- 
ests, with particular reference to the canopies of Doryphora sassafras (Monimiaceae). Biotropica, 24, 263-272. doi:10.2307/2388521

[26] Coley, P.D. and Barone, J.A. (1996) Herbivory and plant defences in tropical forests. Annual Review of Ecology and Systematics, 27, 305-335.

doi:10.1146/annurev.ecolsys.27.1.305

[27] Landsberg, J. and Ohmart, C. (1989) Levels of insect defoliation in forests: Patterns and concepts. Trends in Ecology and Evolution, 4, 96-100. doi:10.1016/0169-5347(89)90054-2

[28] Read, J., Gras, E., Sanson, G.D., Clissold, F. and Brunt, C. (2003) Does chemical defence decline more in developing leaves that become strong and tough at maturity. Australian Journal of Botany, 51, 489-496. doi:10.1071/BT03044

[29] Thaler, J.S. (2002) Effect of jasmonate-induced plant responses on the natural enemies of herbivores. Journal of Animal Ecology, 71, 141-150. doi:10.1046/j.0021-8790.2001.00586.x

[30] Louda, S.M. (1982) Distribution ecology: Variation in plant recruitment over a gradient in relation to insect seed predation. Ecological Monographs, 52, 25-41. doi: $10.2307 / 2937343$

[31] Louda, S.M. (1983) Seed predation and seedling mortality in the recruitment of a shrub, Haplopappus venetus (Asteraceae), along a climatic gradient. Ecology, 64, 511521. doi:10.2307/1939971

[32] Lincoln, D.E. and Mooney, H.A. (1984) Herbivory on Diplacus aurantiacus shrubs in sun and shade. Oecologia, 64, 173-176. doi:10.1007/BF00376867

[33] Koptur, S. (1985) Alternative defenses against herbivores in Inga (Fabaceae: Mimosoideae) over an elevational gradient. Ecology, 66, 1639-1650. doi:10.2307/1938026

[34] Collinge, S.K. and Louda, S.M. (1988) Herbivory by leaf miners in response to experimental shading of a native crucifer. Oecologia, 75, 559-566. doi:10.1007/BF00776420

[35] Morrow, P.A. and Fox, L.R. (1989) Estimates of presettlement insect damage in Australian and North American forests. Ecology, 70, 1055-1060. doi:10.2307/1941374

[36] Galen, C. (1990) Limits to the distributions of alpine tundra plants: Herbivores and the alpine skypilot, Polemonium viscosum. Oikos, 59, 355-358. doi:10.2307/3545146

[37] Louda, S.M. and Collinge, S.K. (1992) Plant resistance to insect herbivores: A field test of the environmental stress hypothesis. Ecology, 73, 153-169. doi:10.2307/1938728

[38] Stone, C. and Bacon, P.E. (1994) Relationships among moisture stress, insect herbivory, fliar cineole content and the growth of river red gum Eucalyptus camaldulensis. Journal of Applied Ecology, 31, 604-612. doi:10.2307/2404151

[39] Aizen, M.A. and William, A.P. (1995) Leaf phenology and herbivory along a temperature gradient: A spatial test of the phenological window hypothesis. Journal of Vegetation Science, 6, 543-550. doi:10.2307/3236353
[40] Landsberg, J. and Gillieson, D.S. (1995) Regional and local variation in insect herbivory, vegetation and soils of eucalypt associations in contrasted landscape positions along a climatic gradient. Australian Journal of Ecology, 20, 299-315. doi:10.1111/j.1442-9993.1995.tb00542.x

[41] Louda, S.M. and Rodman, J.E. (1996) Insect herbivory as a major factor in the shade distribution of a native crucifer (Cardamine cordifolia A. Gray, Bittercress). Journal of Ecology, 84, 229-237. doi:10.2307/2261358

[42] Feller, I.C. and Mathis, W.N. (1997) Primary herbivory by wood-boring insects along an architectural gradient of Rhizophora mangle. Biotropica, 29, 440-451. doi:10.1111/j.1744-7429.1997.tb00038.x

[43] Kelly, C.A. (1998) Effects of variable life history and insect herbivores on reproduction in Solidago macrophylla (Asteraceae) on an elevational gradient. American Midland Naturalist, 139, 243-254. doi:10.1674/0003-0031(1998)139[0243:EOVLHA]2.0.C $\underline{\mathrm{O} ; 2}$

[44] Uriarte, M. and Schmitz, O.J. (1998) Trophic control across a natural productivity gradient with sap-feeding herbivores. Oikos, 82, 552-560. doi:10.2307/3546375

[45] Maron, J.L. (1998) Insect herbivory above- and belowground: Individual and joint effects on plant fitness. Ecology, 79, 1281-1293. doi:10.1890/0012-9658(1998)079[1281:IHAABI]2.0.CO; $\underline{2}$

[46] McEvoy, P.B. and Coombs, E.M. (1999) Biological control of plant invaders: regional patterns, field experiments, and structured population models. Ecological Applications, 9, 387-401. doi:10.1890/1051-0761(1999)009[0387:BCOPIR]2.0.CO ;2

[47] Alonso, C. (1999) Variation in herbivory by Yponomeuta mahalebella on its only host plant Prunus mahaleb along an elevational gradient. Ecological Entomology, 24, 371379. doi:10.1046/j.1365-2311.1999.00211.x

[48] Fagan, W.F. and Bishop, J.G. (2000) Trophic interactions during primary succession: Herbivores slow a plant reinvasion at Mount St. Helens. The American Naturalist, 155, 238-251. doi:10.1086/303320

[49] Pennings, S.C., Siska, E.L. and M.D. Bertness (2001) Latitudinal differences in plant palatability in atlantic coast salt marshes. Ecology, 82, 1344-1359. doi:10.1890/0012-9658(2001)082[1344:LDIPPI]2.0.CO; $\underline{2}$

[50] Rand, T.A. (2002) Variation in insect herbivory across a salt marsh tidal gradient influences plant survival and distribution. Oecologia, 132, 549-558. doi:10.1007/s00442-002-0989-2

[51] Bale, J.S., Masters, G.J., Hodkinson, I.D., Awmack, C., Bezemer, T.M., Brown, V.K., Butterfield, J., Buse, A., Coulson, J.C., Farrar, J., Good, J.E.G., Harrington, R., Hartley, S., Jones, T.H., Lindroth, R.L., Press, M.C., Symrnioudis, I., Watt, A.D. and Whittaker, J.B. (2002) Herbivory in global climate change research: Direct effects of rising temperature on insect herbivores. Global Change Biology, 8, 1-16. 
doi:10.1046/j.1365-2486.2002.00451.X

[52] Freeman, R.S., Alison, K.B. and Neefus, C.D. (2003) Flowering phenology and compensation for herbivory in Ipomopsis aggregata. Oecologia, 136, 394-401. doi:10.1007/s00442-003-1276-6

[53] Hódar, J. and Zamora, R. (2004) Herbivory and climatic warming: A Mediterranean outbreaking caterpillar attacks a relict, boreal pine species. Biodiversity and Conservation, 13, 493-500. doi:10.1023/B:BIOC.0000009495.95589.a7

[54] Cuevas-Reyes, P., Quesada, M., Siebe, C. and Oyama, K. (2004) Spatial patterns of herbivory by gall-forming insects: A test of the soil fertility hypothesis in a Mexican tropical dry forest. Oikos, 107, 181-189. doi:10.1111/j.0030-1299.2004.13263.x

[55] Gaston, K.J., Genney, D.R., Thurlow, M. and Hartley, S.E. (2004) The geographical range structure of the holly leafminer. IV. Effects of variation in host-plant quality. Journal of Animal Ecology, 73, 911-924. doi:10.1111/j.0021-8790.2004.00866.X

[56] Novotny, V. and Basset, Y. (2005) Host specificity of insect herbivores in tropical forests. Proceedings: Biological Sciences, 272, 1083-1090. doi:10.1098/rspb.2004.3023

[57] Knight, T.M. and Holt, R.D. (2005) Fire generates spatial gradients in herbivory: An example from a Florida sandhill ecosystem. Ecology, 86, 587-593. doi:10.1890/04-1069

[58] Pennings, S.C. and Silliman, B.R. (2005) Linking biogeography and community ecology: latitudinal variation in plant-herbivore interaction strength. Ecology, 86, 23102319. doi:10.1890/04-1022

[59] Fagan, W.F., Lewis, M., Neubert, M.G., Aumann, C., Apple, J.L. and Bishop, J.G. (2005) When can herbivores slow or reverse the spread of an invading plant. A test case from Mount St. Helens. The American Naturalist, 166, 669-685. doi:10.1086/497621

[60] Unsicker, S., Baer, N., Kahmen, A., Wagner, M., Buchmann, N. and Weisser, W. (2006) Invertebrate herbivory along a gradient of plant species diversity in extensively managed grasslands. Oecologia, 150, 233. doi:10.1007/s00442-006-0511-3

[61] Siemann, E., Rogers, W.E. and Dewalt, S.J. (2006) Rapid adaptation of insect herbivores to an invasive plant. Proceedings of the Royal Society B: Biological Sciences, 273, 2763-2769. doi:10.1098/rspb.2006.3644

[62] Maron, J.L. and Crone, E. (2006) Herbivory: Effects on plant abundance, distribution and population growth. Proceedings of the Royal Society B: Biological Sciences, 273, 2575-2584. doi:10.1098/rspb.2006.3587

[63] Pennings, S.C., Zimmer, M., Dias, N., Sprung, M., Dave, N., Ho, C.-K., Kunza, A., McFarlin, C., Mews, M., Pfauder, A. and Salgado, C. (2007) Latitudinal variation in plant-herbivore interactions in European salt marshes. Oikos, 116, 543-549. doi:10.1111/j.2007.0030-1299.15591.x

[64] Pontes R. and Basset, Y. (2007) Gall-forming and free- feeding herbivory along vertical gradients in a low-land tropical rainforest: The importance of leaf sclero-phylly. Ecography, 30, 663-672. doi:10.1111/j.2007.0906-7590.05083.x

[65] Kozlov, M. (2008) Losses of birch foliage due to insect herbivory along geographical gradients in Europe: A climate-driven pattern. Climatic Change, 87, 107-117. doi:10.1007/s10584-007-9348-y

[66] Sinclair, R.J. and Hughes, L. (2008) Incidence of leaf mining in different vegetation types across rainfall, canopy cover and latitudinal gradients. Austral Ecology, 33, 353-360. doi:10.1111/j.1442-9993.2007.01825.x

[67] Wolf, A., Kozlov, M.V. and Callaghan, T.V. (2008) Impact of non-outbreak insect damage on vegetation in northern Europe will be greater than expected during a changing climate. Climatic Change, 87, 91-106. doi:10.1007/s10584-007-9340-6

[68] Adams, J.M. and Zhang, Y. (2009) Is there more insect folivory in warmer temperate climates? A latitudinal comparison of insect folivory in eastern North America. Journal of Ecology, 97, 933-940. doi:10.1111/j.1365-2745.2009.01523.x

[69] Adams, J., Rehill, B., Zhang, Y. and Gower, J. (2009) A test of the latitudinal defense hypothesis: Herbivory, tannins and total phenolics in four North American tree species. Ecological Research, 24, 697-704. doi:10.1007/s11284-008-0541-X

[70] Adams, J., Zhang, Y., Basri, M. and Shukor, N. (2009) Do tropical forest leaves suffer more insect herbivory? A comparison of tropical versus temperate herbivory, estimated from leaf litter. Ecological Research, 24, 13811392. doi:10.1007/s11284-009-0623-4

[71] Sobek, S., Scherber, C., Steffan-Dewenter, I. and T. Tscharntke (2009) Sapling herbivory, invertebrate herbivores and predators across a natural tree diversity gradient in Germany's largest connected deciduous forest. Oecologia, 160, 279-288. doi:10.1007/s00442-009-1304-2

[72] Miller, T.E.X., Louda, S.M., Rose, K.A. and Eckberg, J.O. (2009) Impacts of insect herbivory on cactus population dynamics: Experimental demography across an environmental gradient. Ecological Monographs, 79, 155-172. doi:10.1890/07-1550.1

[73] Del-Val, E. and Armesto, J.J. (2010) Seedling mortality and herbivory damage in subtropical and temperate populations: Testing the hypothesis of higher herbivore pressure toward the tropics. Biotropica, 42, 174-179. doi:10.1111/j.1744-7429.2009.00554.x

[74] O’Neill, B., Zangerl, A., Dermody, O., Bilgin, D., Casteel, C., Zavala, J., DeLucia, E. and M. Berenbaum (2010) Impact of elevated levels of atmospheric $\mathrm{CO}_{2}$ and herbivory on flavonoids of soybean (Glycine max Linnaeus). Journal of Chemical Ecology, 36, 35-45. doi:10.1007/s10886-009-9727-0

[75] Schuldt, A., Baruffol, M., Böhnke, M., Bruelheide, H., Härdtle, W., Lang, A.C., Nadrowski, K., Oheimb, G. Von, Voigt, W., Zhou, H. and Assmann T. (2010) Tree diversity promotes insect herbivory in subtropical forests of southeast China. Journal of Ecology, 98, 917-926. 
doi:10.1111/j.1365-2745.2010.01659.x

[76] Ballhorn, D.J., Schmitt, I., Fankhauser, J.D., Katagiri, F. and Pfanz, H. (2011) $\mathrm{CO}_{2}$-mediated changes of plant traits and their effects on herbivores are determined by leaf age. Ecological Entomology, 36, 1-13. doi:10.1111/j.1365-2311.2010.01240.X

[77] Zhang, Y., Adams, J. and Zhao, D. (2011) Does insect folivory vary with latitude among temperate deciduous forests. Ecological Research, 26, 377-383. doi:10.1007/s11284-010-0792-1

[78] Björkman, C., Berggren, Å. and Bylund, H. (2011) Causes behind insect folivory patterns in latitudinal gradients. Journal of Ecology, 99, 367-369.

[79] Cornelissen, T. and Stiling, P. (2011) Similar responses of insect herbivores to leaf fluctuating asymmetry. Arthropod-Plant Interactions, 5, 59-69. doi:10.1007/s11829-010-9116-1

[80] Garibaldi, L., Kitzberger, T. and Chaneton, E. (2011) Environmental and genetic control of insect abundance and herbivory along a forest elevational gradient. Oecologia, 167, 117-129. doi:10.1007/s00442-011-1978-0

[81] Garibaldi, L.A., Kitzberger, T. and Ruggiero, A. (2011) Latitudinal decrease in folivory within Nothofagus pumilio forests: Dual effect of climate on insect density and leaf traits. Global Ecology and Biogeography, 20, 609619. doi:10.1111/j.1466-8238.2010.00623.x

[82] Blue, J., Souza, L., Classen, A., Schweitzer, J. and Sanders, N. (2011) The variable effects of soil nitrogen availability and insect herbivory on aboveground and belowground plant biomass in an old-field ecosystem. Oecologia, 167, 771-780. doi:10.1007/s00442-011-2028-7

[83] Silva, J., Espírito-Santo, M. and Melo, G. (2012) Herbivory on Handroanthus ochraceus (Bignoniaceae) along a successional gradient in a tropical dry forest. ArthropodPlant Interactions, 6, 45-57. doi:10.1007/s11829-011-9160-5 